\title{
Quantum dynamics of a quark-binding bubble in two space and one time dimensions*
}

\author{
R. C. Giles ${ }^{\dagger}$ and S.-H. H. Tye \\ Stanford Linear Accelerator Center, Stanford University, Stanford, California 94305 \\ (Received 12 December 1975)
}

\begin{abstract}
We discover that the set of coupled equations of motion of the quark-binding bubble model can be solved exactly and completely in two space and one time dimensions. In this three-dimensional Minkowski space, the bubble is equivalent to a closed string with massless quarks trapped on it. The integrability of the equations follows from the special simplicity of the geometry of two-dimensional surfaces. From the set of all classical solutions, a Poincaré-invariant quantum system is explicitly constructed. The resulting spectrum is free of ghosts.
\end{abstract}

\section{INTRODUCTION}

Recently several dynamical models of quark binding within hadrons have been proposed. In particular, Bardeen, Chanowitz, Drell, Weinstein, and $\mathrm{Yan}^{1}$ (BCDWY) proposed a field-theoretic model where quarks are bound on the surface of the bubble. In a classical picture, the bubble surface arises as a domain boundary of a region where a scalar field assumes the "wrong" vacuum expectation value. In the preceding paper,,$^{2}$ one of us (R.G.) has reformulated the BCDWY model in the zero-thickness (of the bubble surface) limit. In this formulation, the scalar field is replaced as a dynamical variable by the geometry of the bubble surface, and the quark degrees of freedom are characterized completely by a surface quark field. Although the quantum theory can be derived from an action principle, quantum corrections of the original field-theoretic description become intractable; still the action formulation has the advantage of allowing ready calculations of many properties of the system.

In this work we concentrate on the bubble in three spacetime dimensions. We shall see that all classical solutions to the general, time-dependent bubble theory can be constructed explicitly. In the general case, the bubble executes a complicated, but periodic, oscillation in time. The quarks trapped on it are massless and move along lightlike lines embedded in the surface. There is a degeneracy over an infinite class of shapes of the bubble. By choosing a special coordinate system and a particular Lorentz frame, we can represent all possible solutions to the theory in terms of a countable number of independent normal-mode amplitudes. We exhibit a set of commutation relations among these modes which provide a Poincaré-invariant quantum theory of the single bubble.

The bubble in three spacetime dimensions is equivalent to a closed string upon which quarks are trapped; our method of solution closely resembles that of the Nambu action. ${ }^{3}$ In the absence of quark fields, the spectrum reduces to that of the closed string.

In the presence of quark fields, the bubble equations of motion form a rather complicated set of coupled nonlinear partial differential equations. They are difficult to solve directly. We proceed as follows: First, exploiting some special geometric properties of two-dimensional surfaces and rather general properties of the equations of motion, we show that coordinates may be chosen in which the equation of motion of the bubble surface has a particularly simple form. Using this result, we find that the Dirac equation can be solved to give the Dirac field everywhere on the surface in terms of the dynamical variables describing the surface geometry. Finally, we show that the surface equation of motion gives an algebraic relation between the Dirac field and the surface variables. The complete quantized spectrum can then be exhibited. Since the solution is obtained by breaking manifest Lorentz covariance, all that remains to be shown is the straightforward demonstration of the closure of the Poincaré algebra.

Classically the bubble energy is proportional to its length, ${ }^{2}$ independent of its shape. In the quantized system this property is reflected by a Hagedorn-like spectrum (where the number of states increases exponentially with the mass), with linearly rising Regge trajectories. The Regge slope is inversely proportional to the bubble constant $\mathfrak{C}$ and the intercept of the leading Regge trajectory must be an integer or a half integer. For some choices of the intercept, it takes a negative amount of energy to generate a bubble. This is in contrast to the classical or semiclassical situation where generating a bubble always takes a positive amount of energy. This particular quantum property may allow the avoidance of the apparent problem of bubble condensation.

The rest of this work is organized as follows: 
Sec. II contains the formulation of the bubble system. For more details of the differential geometry and the derivations, the reader is referred to the preceding paper. In Sec. III, we derive some of the properties of the bubble which are necessary for the solution. In particular, reparametrization invariance allows us to choose a gauge such that the bubble equation of motion becomes very simple. The fermion field can then be solved in terms of the geometry. The solution is presented in Sec. IV and the results in Sec. V. In Sec. VI we discuss among other things the relation of this three-dimensional bubble to other extended-hadron models.

\section{BUBBLE MODEL}

The bubble of interest is a two-dimensional hypersurface embedded in a three-dimensional Minkowski space. The bubble surface (Fig. 1)

$$
x_{\mu}=R_{\mu}\left(u^{\alpha}\right), \quad \mu=0,1,2 ; \alpha=0,1
$$

is parametrized by the internal coordinates $u^{\alpha}$, and is assumed to be a differentiable manifold.

The metric in Minkowski space $\eta_{\mu \nu}$ induces a metric on the hypersurface

$$
g_{\alpha \beta}=\eta_{\mu \nu} \tau_{\alpha}^{\mu} \tau_{\beta}^{\nu}=\tau_{\alpha} \cdot \tau_{\beta},
$$

where $\tau_{\alpha}^{\mu}=\partial R^{\mu} / \partial u^{\alpha} \equiv \partial_{\alpha} R^{\mu} \equiv R_{\mid \alpha}^{\mu}$ are the tangent vectors. At each point on the surface, a unique spacelike unit outward normal vector $n_{\mu}\left(u^{\alpha}\right)$ can
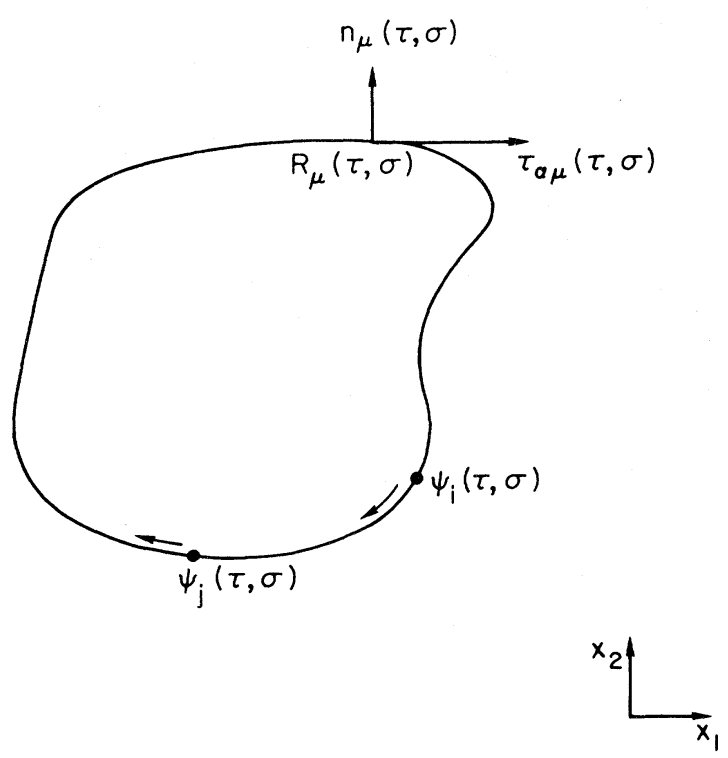

FIG. 1. A quark-confining bubble in two space dimensions and one time dimension. be defined

$$
\begin{aligned}
& n \cdot \tau_{\alpha}=0, \\
& n^{2}=n \cdot n=-1 .
\end{aligned}
$$

It is useful to express $\eta_{\mu \nu}$ at each point of the surface in terms of the set of vectors at the point

$$
\eta_{\mu \nu}=\tau_{\mu}^{\beta} \tau_{\beta \nu}-n_{\mu} n_{\nu}
$$

Another useful tensor is the curvature tensor

$$
h_{\alpha \beta}=n_{\mid \alpha} \cdot \tau_{\beta}=-n \cdot \tau_{\alpha \mid \beta}=-n \cdot \tau_{\beta \mid \alpha} .
$$

Its trace $h_{\alpha}^{\alpha}=2 k$ is proportional to the local mean curvature of the surface. The action of the bubble is

$$
\begin{aligned}
S & =\int d^{2} u^{\alpha} \mathcal{L} \\
& =\int d u^{0} d u^{1} \sqrt{|g|}\left[i \bar{\psi}_{j}\left(t^{\alpha} \partial_{\alpha}+k \eta\right) \psi_{j}-\mathfrak{C}\right],
\end{aligned}
$$

where $g$ is the determinant of the metric tensor $g_{\alpha \beta} . \quad \psi_{j}$ is an arbitrary spinor field satisfying the constraint $i h \psi_{j}=\psi_{j}$, where $h=n_{\mu} \gamma^{\mu} ; \gamma_{\mu}$ are the Dirac matrices. This constraint ensures that the fermion current density and the energy-momentum density are tangential. For example,

$$
n_{\mu} \bar{\psi} \gamma^{\mu} \psi=\bar{\psi} h \psi=-i \bar{\psi} \psi=i \bar{\psi} \psi=0 .
$$

The index $j$ designates the quark species. For simplicity we shall consider only one type of quark field. The generalization to many types is straightforward. $\mathcal{C}$ is a constant and provides the only scale of the system.

The Euler-Lagrange equation

$$
\frac{\delta \mathscr{L}}{\delta \bar{\psi}}=\partial_{\alpha} \frac{\delta \mathscr{L}}{\delta \partial_{\alpha} \bar{\psi}}
$$

gives the Dirac equation

$$
i\left(t^{\alpha} \partial_{\alpha}+k h\right) \psi=0 \text {. }
$$

Using this and the constraint

$$
i h \psi=\psi,
$$

we obtain, from the Euler-Lagrange equation,

$$
n^{\mu} \partial_{\alpha} \frac{\delta \mathscr{L}}{\delta \tau_{\alpha}^{\mu}}=0,
$$

the surface equation of motion

$$
h_{\alpha \beta} T^{\alpha \beta}=0 \text {, }
$$

where the energy-momentum tensor $T^{\alpha \beta}$ is given by

$$
\begin{aligned}
T^{\alpha \beta} & =\mathfrak{e} g^{\alpha \beta}+\theta^{\alpha \beta} \\
& =\mathfrak{e} g^{\alpha \beta}+\frac{i}{2} \bar{\psi} t^{\alpha} \ddot{\partial}^{\beta} \psi,
\end{aligned}
$$


where $\bar{\psi} t^{\alpha} \ddot{\partial}^{\beta} \psi=\bar{\psi} t^{\alpha} \partial^{\beta} \psi-\left(\partial^{\beta} \bar{\psi}\right) t^{\alpha} \psi$. Equations (1), along with the global requirement that the surface be spatially closed define the bubble system completely. Equation (1c) describes the vibrations of the surface. It is the normal component of the surface Euler-Lagrange equation. Equations (1a), $(1 \mathrm{~b})$, and (1c) form a set of coupled equations. The tangential components of the surface Euler-Lagrange equation

$$
\tau_{\beta}^{\mu}\left(\partial_{\alpha} \frac{\delta \mathscr{L}}{\delta \tau_{\alpha}^{\mu}}\right)=0
$$

give

$$
\begin{aligned}
T^{\alpha \beta}{ }_{1 \alpha} & =T^{\alpha \beta}{ }_{1 \alpha}+\left\{\begin{array}{c}
\beta \\
\alpha \gamma
\end{array}\right\} T^{\alpha \gamma}+\left\{\begin{array}{c}
\alpha \\
\alpha \gamma
\end{array}\right\} T^{\gamma \beta} \\
& =0 .
\end{aligned}
$$

It is straightforward to show that this follows from Eqs. (1a)-(1d) and, hence, not another independent equation of motion. This is a reflection of gauge invariance under coordinate transformation. $\left\{\begin{array}{c}\alpha \\ \beta \gamma\end{array}\right\}$ is the Christoffel symbol of the second kind and is given by

$$
\begin{aligned}
\left\{\begin{array}{c}
\alpha \\
\beta \gamma
\end{array}\right\} & =\frac{1}{2} g^{\alpha \sigma}\left(-g_{\beta \gamma \mid \delta}+g_{\beta \delta \mid \gamma}+g_{\sigma \gamma \mid \beta}\right) \\
& =\tau^{\alpha} \cdot \tau_{\beta \mid \gamma}
\end{aligned}
$$

In the three spacetime dimensions, both the geometry of the bubble and the $\gamma$-matrix algebra can be simplified. The bubble surface is two dimensional. Our notation will be

$$
R_{\mu}\left(u^{0}, u^{1}\right)=R_{\mu}(\tau, \sigma), \quad u^{0} \equiv \tau, \quad u^{1} \equiv \sigma,
$$

with

$$
\dot{A}=\frac{\partial A}{\partial \tau}, \quad A^{\prime}=\frac{\partial A}{\partial \sigma}
$$

for any quantity $A$. We choose the orientation of the internal coordinates such that

$$
n^{\mu}=-\frac{1}{\sqrt{-g}} \epsilon^{\mu \nu \lambda}\left(\tau_{0}\right)_{\nu}\left(\tau_{1}\right)_{\lambda} .
$$

In three dimensions, we need only three matrices satisfying the anticommutation relations

$$
\left\{\gamma^{\mu}, \gamma^{\nu}\right\}=2 \eta^{\mu \nu}, \quad \mu, \nu=0,1,2 \text {. }
$$

We choose these to be $2 \times 2$ Pauli matrices rather than the usual $4 \times 4 \gamma$ matrices

$$
\gamma^{0}=\sigma_{1}, \quad \gamma^{1}=i \sigma_{3}, \quad \gamma^{2}=-i \sigma_{2},
$$

whose algebra is

$$
\gamma^{\mu} \gamma^{\nu}=\eta^{\mu \nu}-i \epsilon^{\mu \nu \lambda} \gamma_{\lambda} .
$$

That such a choice is possible is obvious mathematically. Its significance in the theory becomes clear if we begin with a $4 \times 4$ representation of the usual $\gamma$ matrices

$$
\begin{aligned}
& \gamma^{0}=\left(\begin{array}{cc}
\sigma_{1} & 0 \\
0 & -\sigma_{1}
\end{array}\right), \gamma^{1}=\left(\begin{array}{cc}
i \sigma_{3} & 0 \\
0 & -i \sigma_{3}
\end{array}\right), \\
& \gamma^{2}=\left(\begin{array}{cc}
-i \sigma_{2} & 0 \\
0 & i \sigma_{2}
\end{array}\right), \quad \gamma^{3}=\left(\begin{array}{cc}
0 & 1 \\
-1 & 0
\end{array}\right), \\
& \gamma^{5}=\left(\begin{array}{ll}
0 & 1 \\
1 & 0
\end{array}\right), \quad \psi=\left(\begin{array}{l}
\psi_{+} \\
\psi_{-}
\end{array}\right) .
\end{aligned}
$$

In this representation, the Dirac equation involves only $\gamma^{0}, \gamma^{1}, \gamma^{2}$. Thus, the two-component spinors $\psi_{+}$and $\psi_{-}$decouple from each other. Because the fermion moves in a single plane, there is an extra conserved "charge" whose matrix is $\gamma^{3} \gamma_{5}$. To choose a two-component representation of the $\gamma$ matrices is to impose the condition that the Dirac field be an eigenstate of $\gamma^{3} \gamma_{5}$ with eigenvalue +1 .

The theory we obtain by making this choice is a consistent and complete theory of a fermion trapped on the bubble surface. That this is so is not completely obvious. In a three-dimensional theory where the fermion is free to move throughout space-time, charge-conjugation and time-reversal invariance cannot be realized in the two-component representation. For example, charge conjugation must be represented by a matrix $C$ with the property

$$
C \gamma^{\mu *} C^{-1}=\gamma^{\mu} \text {. }
$$

In the representation (4), we must take $C=\gamma_{5} \gamma^{1}$. Thus, $C$ does not commute with $\gamma^{3} \gamma_{5}$. Because the fermion in the bubble theory is confined to the two-dimensional bubble surface, however, the requirement (5) need be satisfied only by the tangential components of the $\gamma$ matrices:

$$
C t_{\alpha}^{*} C^{-1}=t_{\alpha} \text {. }
$$

This condition can be realized in the two-component representation by $C=i \hbar \gamma^{1}$. The two-component representation is "complete" for the bubble theory in the sense that $P, C$, and $T$ can all be realized for the Fermi field.

We must also emphasize that a bubble theory based on a four-component Dirac spinor is not an altogether trivial extension of the two-component theory. It may be viewed as a theory of two independent two-component quark fields, $\psi_{+}$and $\psi_{-}$, trapped on the bubble surface. However, the twocomponent version of Eq. (1b) is different for these two spinors:

$$
\begin{aligned}
& i n \psi_{+}=\psi_{+}, \\
& i n \psi_{-}=-\psi_{-} .
\end{aligned}
$$

Though the Dirac equations for these two spinors 
separate completely, both interact with the surface through Eq. (1c). Because of the difference in sign in Eq. (6), the effects on the surface of the two spinor fields do not add in a simple way. In this paper we only examine the two-component case.

\section{PROPERTIES OF THE BUBBLE}

To solve Eq. (1) we first have to obtain some useful properties of the bubble system. Because the system is invariant under arbitrary coordinate transformations

$$
u^{\alpha} \rightarrow f^{\alpha}\left(u^{\beta}\right) \text {, }
$$

we are at liberty to choose a system of coordinates which simplify the equations. Further, as we shall see below, the requirement that a solution to the equations of motion exist at all places very strong constraints on the geometric structure of the surface. These constraints arise, essentially, from the causal structure of the free, massless Dirac field on the surface.

We shall show that the equations of motion imply there exists a coordinate system such that

$$
\dot{R}_{\mu}^{\prime}(\tau, \sigma)=0
$$

and

$$
R^{\prime 2}=R_{\mu}^{\prime} \cdot R^{\mu \prime}=0
$$

A special property of two-dimensional manifolds which we rely on to choose coordinates is that any symmetric tensor of signature $(1,-1)$ can be brought into off-diagonal form by a coordinate transformation. This would, for example, allow us to choose the metric to be off-diagonal. It is more useful, however, to work in coordinates where the stress tensor $T^{\alpha \beta}$ is off-diagonal. To do this, we have to show that the stress tensor

$$
\begin{aligned}
T^{\alpha \beta} & =\mathfrak{e} g^{\alpha \beta}-\operatorname{Im}\left(\bar{\psi} t^{\alpha} \partial^{\beta} \psi\right) \\
& =\mathfrak{e} g^{\alpha \beta}+\theta^{\alpha \beta}
\end{aligned}
$$

is symmetric and has signature $(1,-1)$.

First, we show that if $T^{\alpha \beta}$ is any symmetric tensor with signature $(1,-1)$ on a two-dimensional Riemann manifold, then a local coordinate system can be found in which $T^{\alpha \beta}$ is off-diagonal: $T^{00}=T^{11}=0$. Let $\left(v^{0}, v^{1}\right)$ be the desired coordinates, and $\tilde{T}^{\gamma \delta}$ be the tensor $T^{\alpha \beta}$ in these coordinates:

$$
\tilde{T}^{\gamma 6}=T^{\alpha \beta} \frac{\partial v^{\gamma}}{\partial u^{\alpha}} \frac{\partial v^{6}}{\partial u^{\beta}} .
$$

We require

$$
\begin{aligned}
& \tilde{T}^{00}=T^{\alpha \beta} \frac{\partial v^{0}}{\partial u^{\alpha}} \frac{\partial v^{0}}{\partial u^{\beta}}=0, \\
& \tilde{T}^{11}=T^{\alpha \beta} \frac{\partial v^{1}}{\partial u^{\alpha}} \frac{\partial v^{1}}{\partial u^{\beta}}=0 .
\end{aligned}
$$

Both $v^{0}$ and $v^{1}$ must have gradients which satisfy the homogeneous quadratic constraint (9a). Because $T^{\alpha \beta}$ has signature $(1,-1)$ the solutions of (9a) are such that the gradient must lie on a degenerate hyperbola (analogous to the light cone) in the tangent space to the surface at each point. There are two independent real solutions to the quadratic equation at each point. These generate two functionally independent solutions to the differential equation, which can be taken to be $v^{0}$ and $v^{1}$.

Next, we must show that the energy-momentum tensor $T^{\alpha \beta}$ satisfies the conditions for this theorem: that $T^{\alpha \beta}$ is symmetric and has signature $(1,-1)$.

The metric tensor is symmetric, so the symmetry of $T^{\alpha \beta}$ will follow if we show that $\theta^{\alpha \beta}$ is symmetric. It is sufficient to show that $\theta^{\alpha \beta}$ is symmetric at any given point in some coordinate system. A tensor which is symmetric at a point in one coordinate system is symmetric at that point in all coordinate systems. At a given point, $u^{\alpha}$, we choose locally geodesic coordinates:

$$
\begin{aligned}
& g_{\alpha \beta}=\left(\begin{array}{cc}
1 & 0 \\
0 & -1
\end{array}\right), \\
& g_{\alpha \beta \mid \gamma}=0 .
\end{aligned}
$$

We want to show that $\theta^{01}=\theta^{10}$ at the point $u^{\alpha}$. We have

$$
\theta^{01}-\theta^{10}=-\operatorname{Im} \bar{\psi}\left(t^{0} \partial^{1}-\not^{1} \partial^{0}\right) \psi .
$$

Using the Dirac equation

$$
\left(i t^{0} \partial_{0}+i t^{1} \partial_{1}+k\right) \psi=0
$$

and the relation, valid in the two-component representation of the $\gamma$ matrices, following from (9b)

$$
t^{1} f^{0}=-i n
$$

we have

$$
\partial_{1} \psi=\left(-i \not h \partial_{0}-i k t^{1}\right) \psi \text {. }
$$

Then (9c) becomes

$$
\theta^{01}-\theta^{10}=-\operatorname{Im} \bar{\psi}\left[-\left(t^{0} i n+t^{1}\right) \partial_{0}+i k f^{0} t^{1}\right] \psi ;
$$

but

$$
\begin{aligned}
& t^{0} t^{1}=i \hbar, \\
& \bar{\psi}(-i n) \psi=0, \\
& f^{0} i \hbar=-t_{1}=t^{1} .
\end{aligned}
$$

So $\theta^{01}=\theta^{10}$ at the point $u^{\alpha}$. Therefore, $\theta^{\alpha \beta}$ is symmetric at all points in all coordinate systems.

Finally, we must show that $T^{\alpha \beta}$ has signature $(1,-1)$. It is sufficient to show that $\operatorname{det}\left(T^{\alpha \beta}\right)<0$, for then the eigenvalues of $T^{\alpha \beta}$ have opposite sign. Because $T^{\alpha \beta}$ is a $2 \times 2$ matrix, we have 


$$
\operatorname{det}\left(T^{\alpha \beta}\right)=\frac{\mathfrak{e}^{2}}{g}+\frac{\mathfrak{e}}{g} g_{\alpha \beta} \theta^{\alpha \beta}+\operatorname{det}\left(\theta^{\alpha \beta}\right) .
$$

From the Dirac equation, $g_{\alpha \beta} \theta^{\alpha \beta}=0$. We can also show that $\operatorname{det}\left(\theta^{\alpha \beta}\right)=0$. As will be proved below, the Dirac current, $J_{\alpha}=\bar{\psi} t_{\alpha} \psi$, is lightlike and satisfies

$$
J_{\alpha} \theta^{\alpha \beta}=0 \text {. }
$$

It follows immediately that $\theta^{\alpha \beta}$ is of the form

$$
\theta^{\alpha \beta}=\Lambda J^{\alpha} J^{\beta},
$$

where $\Lambda$ is some scalar function. Then

$$
\operatorname{det}\left(\theta^{\alpha \beta}\right)=\Lambda^{2}\left[\left(J^{0}\right)^{2}\left(J^{1}\right)^{2}-\left(J^{0} J^{1}\right)^{2}\right]=0,
$$

so

$$
\operatorname{det}\left(T^{\alpha \beta}\right)=\frac{\mathfrak{e}^{2}}{g}<0,
$$

since

$$
g<0 \text {. }
$$

The symmetry of $T^{\alpha \beta}$ reflects the absence of spin in two dimensions. In general, a spin-dependent divergence must be added to the canonical stress tensor to form the symmetric improved stress tensor. In two dimensions, however, the canonical fermion stress tensor is already "improved."

We choose coordinates such that

$$
T^{\alpha \beta}=\frac{\mathfrak{e}}{T}\left(\begin{array}{ll}
0 & 1 \\
1 & 0
\end{array}\right)
$$

where $T(\tau, \sigma)$ depends on the details of the solution. The coordinate system is not uniquely determined by the condition (10). We still have conformal invariance: (10) is invariant under coordinate transformations of the form

$$
\begin{aligned}
& \tau \rightarrow f(\tau), \\
& \sigma \rightarrow g(\sigma) .
\end{aligned}
$$

So far, we have used nothing but the coordinate invariance of our description of the bubble surface. We now show that a necessary condition for the field equations to be solvable is that $R^{\mu}(\tau, \sigma)$ satisfy (7) and (8).

We begin by considering the algebraic relations between the fermion current $J^{\alpha}$ and the stress tensor $T^{\alpha \beta}$. A result which follows from the twocomponent representation of the $\gamma$ matrices is the following.

If $\psi$ is any spinor satisfying $\psi \psi=0$, then

$$
\left(\bar{\psi} \gamma^{\mu} \psi\right) \gamma_{\mu} \psi=0 \text {. }
$$

Proof: For any two-component spinor, $\psi$, there exists a unique real unit vector, $\hat{m}$, such that

$$
\hat{m} \cdot \vec{\sigma} \psi=\psi \text {. }
$$

Using the $2 \times 2$ representation of the $\gamma$ matrices (3) we have

$$
\begin{aligned}
& \bar{\psi} \gamma^{\mu} \psi=\psi^{\dagger} \psi\left(1, \hat{m}_{2}, \hat{m}_{3}\right), \\
& \bar{\psi} \psi=\left(\psi^{\dagger} \psi\right) \hat{m}_{1}=0,
\end{aligned}
$$

then

$$
\begin{aligned}
\left(\bar{\psi} \gamma^{\mu} \psi\right) \gamma_{\mu} \psi & =\psi^{\dagger} \psi\left(\sigma_{1}-i \hat{m}_{2} \sigma_{3}+i \hat{m}_{3} \sigma_{2}\right) \psi \\
& =\psi^{\dagger} \psi \sigma_{1}(1-\hat{m} \cdot \vec{\sigma}) \psi \\
& =0 .
\end{aligned}
$$

This has the immediate consequences

$$
\begin{aligned}
& J^{\alpha} J_{\alpha}=0, \\
& J_{\alpha} T^{\alpha \beta}=\mathfrak{e} J^{\beta}, \\
& J_{\alpha} J_{\beta} T^{\alpha \beta}=0,
\end{aligned}
$$

where

$$
J^{\alpha}=\bar{\psi} t^{\alpha} \psi \text {. }
$$

Assuming that $J^{\alpha}$ is not identically zero, these relations allow us to determine some components of the metric tensor in terms of $T$.

With the stress tensor of the form (10), Eq. (14) implies $J_{0} J_{1}=0$. We shall see below that the choice of orientation (2) and the condition (1b) require that $J_{1}=0$. Putting this result in (13) and comparing both sides, we find

$$
g^{00}=0, g^{01}=1 / T .
$$

So the metric has the form

$$
g_{\alpha \beta}=\left(\begin{array}{cc}
A T^{2} & T \\
T & 0
\end{array}\right), \quad g^{\alpha \beta}=\left(\begin{array}{cc}
0 & 1 / T \\
1 / T & -A
\end{array}\right), \quad \sqrt{-g}=T,
$$

where $A$ is not determined by this analysis. We note that

$$
0=g_{11}=R^{\prime}(\tau, \sigma)^{2} .
$$

This is condition (8).

The stress tensor is divergenceless [Eq. (1e)]

$$
\begin{aligned}
T^{\alpha \beta}{ }_{1 \alpha}=0 & =\frac{1}{\sqrt{-g}}\left(\sqrt{-g} T^{\alpha \beta}\right)_{\mid \alpha}+\left\{\begin{array}{l}
\beta \\
\gamma 6
\end{array}\right\} T^{\gamma \sigma} \\
& =\frac{1}{T}\left(T T^{\alpha \beta}\right)_{\mid \alpha}+\frac{2 \mathfrak{C}}{T}\left\{\begin{array}{c}
\beta \\
0_{1}
\end{array}\right\} .
\end{aligned}
$$

But $T T^{\alpha \beta}$ is a constant, so we have

$$
\left\{\begin{array}{c}
\beta \\
01
\end{array}\right\}=0, \beta=0,1 .
$$

The equation of motion (1c) of the surface gives

$$
h_{01}=0 \text {. }
$$

The condition (7) now follows immediately:

$$
\dot{R}_{\mu}^{\prime}(\tau, \sigma)=\tau_{\mu 0 \mid 1}=h_{01} n_{\mu}+\left\{\begin{array}{c}
\beta \\
01
\end{array}\right\} \tau_{\beta \mu}=0 \text {. }
$$

We now turn the problem around. Starting with a 
coordinate system satisfying (7) and (8), we derive the solutions to the bubble equations. Equations (7) implies the surface is of the form

$$
R_{\mu}(\tau, \sigma)=Q_{\mu}(\tau)+S_{\mu}(\sigma) .
$$

Defining,

$$
q^{\mu}(\tau)=\tau_{0}^{\mu}=\dot{Q}^{\mu}, \quad s^{\mu}(\sigma)=\tau_{1}^{\mu}=S^{\mu},
$$

we have

$$
s^{2}=0
$$

where

$$
\begin{aligned}
& g_{\alpha \beta}=\left(\begin{array}{cc}
q^{2} & q \cdot s \\
q \cdot s & 0
\end{array}\right), \\
& g^{\alpha \beta}=\left(\begin{array}{cc}
0 & 1 / q \cdot s \\
1 / q \cdot s & -q^{2} /(q \cdot s)^{2}
\end{array}\right), \\
& \tau_{\mu}^{0}=\frac{s_{\mu}}{q \cdot s}, \quad \tau_{\mu}^{1}=\frac{q_{\mu}}{q \cdot s}-\frac{q^{2}}{(q \cdot s)^{2}} s_{\mu}, \\
& n^{\mu}=-\frac{1}{q \cdot s} \epsilon^{\mu \nu \lambda} q_{\nu} s_{\lambda} .
\end{aligned}
$$

In the following discussion, we assume that $q^{2}>0$. In fact, we shall see that the equations of motion imply that $q^{2}$ is proportional to the fermion energy density, which, as a classical function, is not positive definite. We shall proceed as if the energy density is positive, and we shall find that our solutions are self-consistent in the quantum theory after Fermi statistics and normal ordering are taken into account.

So far, our analysis of the surface geometry has been local. The global condition that the surface $R_{\mu}$ be a closed hypertube in spacetime places further constraints on $Q_{\mu}$ and $S_{\mu}$. Geometrically, Eq. (15) asserts that the hypertube is a surface that is swept out by moving a rigid lightlike curve, $S_{\mu}(\sigma)$, along some timelike curve $Q_{\mu}(\tau)$. At each point on the two-dimensional surface, there are but two lightlike directions. Because the hypertube is closed, the lightlike curve $S_{\mu}$ contained in it must spiral up the tube, intersecting $Q_{\mu}$ infinitely many times (Fig. 2). It is clear that, if the surface is to be swept out by the motion of $S_{\mu}$ along $Q_{\mu}$, each of these points of intersection must be equivalent geometrically, except for an over-all timelike translation, $\Lambda_{\mu}$. After choosing appropriate coordinates $\tau$ and $\sigma$ from one interval to the next, we clearly have the result that $Q_{\mu}(\tau)$ and $S_{\mu}(\sigma)$ are semiperiodic functions:

$$
\begin{aligned}
& Q_{\mu}\left(\tau+\tau_{0}\right)=Q_{\mu}(\tau)+\Lambda_{\mu}, \\
& S_{\mu}\left(\sigma+\sigma_{0}\right)=S_{\mu}(\sigma)+\Lambda_{\mu},
\end{aligned}
$$

where $\tau_{0}, \sigma_{0}=$ fixed period, and $\Lambda_{\mu}=$ a constant

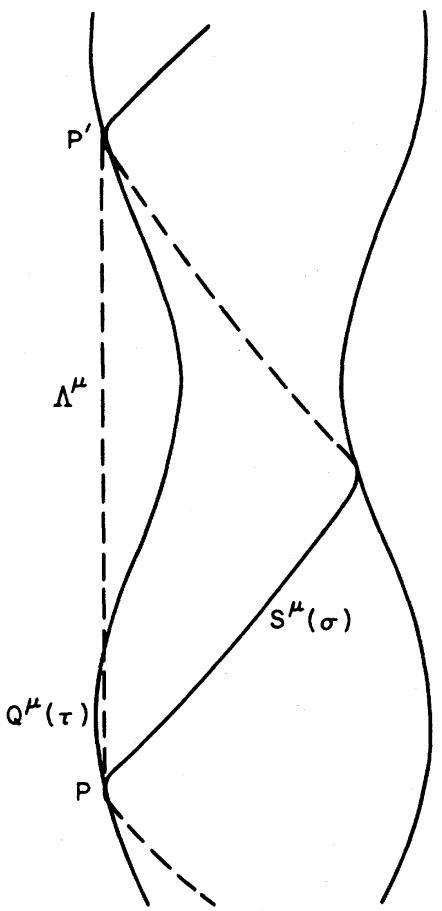

FIG. 2. A three-dimensional bubble generated by curves $Q_{\mu}(\tau)$ and $S_{\mu}(\sigma)$. Points $P$ and $P^{\prime}$ are equivalent.

timelike translation. From this analysis, it is clear that the coordinates $(\tau, \sigma)$ and $\left(\tau+\tau_{0}, \sigma-\sigma_{0}\right)$ correspond to the same point on the hypertube. Later, we will choose ranges for $\tau$ and $\sigma$ so as to bring the coordinate manifold into one-to-one correspondence with the points of the surface.

We proceed to solve the surface Dirac equation in terms of the coordinates (15). The two-component Dirac field has only one complex degree of freedom by virtue of Eq. (1b). Using (2) and (17) we find

$$
\$ q=t_{1} t_{0}=q \cdot s(1+i n) .
$$

We can rewrite (1b) in the equivalent form

$$
\$(\sigma) \psi(\tau, \sigma)=0 \text {. }
$$

The Dirac equation becomes

$$
t_{1}\left(i t^{0} \partial_{0}+i t^{1} \partial_{1}+k\right) \psi=0
$$

or

$$
(1+i n) \psi^{\prime}=0
$$

or

$$
\psi^{\prime}=\frac{i}{2} h^{\prime} \psi=\frac{i}{2} \frac{\epsilon_{\mu \nu \lambda} q^{\mu} s^{\nu} s^{\prime \lambda}}{(q \cdot s)^{2}} q \psi .
$$

Because is independent of $\sigma$, this may be integrated directly: 


$$
\psi(\tau, \sigma)=\exp \left[\frac{i}{2} \int_{0}^{\sigma} d \sigma_{1} \frac{\epsilon_{\mu \nu \lambda} q^{\mu}(\tau) s^{\nu}\left(\sigma_{1}\right) s^{\prime \lambda}\left(\sigma_{1}\right)}{\left[q(\tau) \cdot s\left(\sigma_{1}\right)\right]^{2}} q(\tau)\right] \psi(\tau, 0) .
$$

Given initial data, $\psi(\tau, 0)$, Eq. (21) propagates $\psi$ away from the curve $\sigma=0$, along a family of parallel lightlike lines. The initial data are not entirely free of constraints. First, Eq. (19) must be satisfied:

$$
\$(0) \psi(\tau, 0)=0 \text {. }
$$

Also, because the points $\left(\tau, \sigma_{0}\right)$ and $\left(\tau+\tau_{0}, 0\right)$ are the same, $\psi(\tau, 0)$ must satisfy the periodicity condition:

$$
\psi\left(\tau+\tau_{0}, 0\right)=\exp \left[\frac{i}{2} \int_{0}^{\sigma_{0}} d \sigma_{1} \frac{\epsilon_{\mu \nu \lambda} q^{\mu}(\tau) s^{\nu}\left(\sigma_{1}\right) s^{\prime \lambda}\left(\sigma_{1}\right)}{\left[q(\tau) \cdot s\left(\sigma_{1}\right)\right]^{2}} q(\tau)\right] \psi(\tau, 0) .
$$

The phase integral in (22) is Lorentz invariant, and can most easily be evaluated, for a given $\tau$, in a Lorentz frame where $q_{\mu}=\left(\left(q^{2}\right)^{1 / 2}, \overrightarrow{0}\right)$. We find

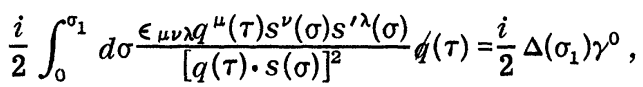

where $\Delta\left(\sigma_{1}\right)$ is the angle through which the spatial part of $s_{\mu}$ has rotated as $\sigma$ varies from 0 to $\sigma_{1}$. Over a full period $\sigma_{0}$, this angle is $2 \pi$, so (22) becomes

$$
\psi\left(\tau+\tau_{0}, 0\right)=-\psi(\tau, 0) .
$$

Thus, $\psi(\tau, 0)$ must be antiperiodic with period $\tau_{0}$.

The physical and geometric interpretation of these solutions to the Dirac equation is clear. The Dirac field is parallel-transported up the surface along the lightlike curves $S_{\mu}$. This is simply the motion of a free massless fermion trapped on a curved surface. On the hypertube, there are two disconnected families of lightlike lines, which spiral up the surface in either the left-handed or the right-handed sense. The condition (1b), in the two-component representation, ensures that the orbits of all quarks in the bubble surface have the same handedness. We note that, by Eq. (6), a bubble theory based on four-component spinors contains both left- and right-handed quarks. This is the reason that the structure of the four-component theory is rather more complicated. As in the static case,$^{2}$ parallel transport once around the tube gives a phase factor of -1 .

We can now understand qualitatively how the causal structure of the Dirac equation induces the periodicity of the surface motion. The Dirac field energy propagates along lightlike curves. These curves must wrap around the surface over and over again. Thus, the initial distribution of Dirac field energy must be reconstructed after the lightlike curves have come once around the bubble. As we have seen generally above and shall see explicitly below, to the extent that the surface is determined by the quark energy distribution, the surface motion is then forced to be periodic.

We now consider the explicit form of Eq. (1c) in terms of $q_{\mu}(\tau), s_{\mu}(\sigma)$, and $\psi(\tau, 0)$. From Eq. (19) and (20) we find that the only nonzero component of the fermion stress tensor is

$$
\theta^{11}=-\frac{1}{(q \cdot s)^{2}} \operatorname{Im} \bar{\psi}(\tau, \sigma) d(\tau) \dot{\psi}(\tau, \sigma) .
$$

Using (21), we can show after some algebra that this is the same as

$$
\theta^{11}=-\frac{1}{(q \cdot s)^{2}} \operatorname{Im} \bar{\psi}(\tau, 0) d(\tau) \dot{\psi}(\tau, 0) .
$$

Then Eq. (1c) is

$$
0=h_{11} T^{11}=h_{11}\left[\theta^{11}-\frac{e q^{2}}{(q \cdot s)^{2}}\right] .
$$

Thus, the equation of motion for the surface is

$$
\mathfrak{e} q^{2}=-\operatorname{Im} \bar{\psi}(\tau, 0) \mathfrak{q}(\tau) \dot{\psi}(\tau, 0) \text {. }
$$

Let us summarize what we have obtained. The surface is described by two periodic vector fields, $Q_{\mu}, S_{\mu}$. The Dirac field is specified by the antiperiodic function, $\psi(\tau, 0)$. The conditions these functions must satisfy in order that they give a solution to the theory are

$$
\begin{aligned}
& q_{\mu}\left(\tau+\tau_{0}\right)=q_{\mu}(\tau), \\
& s_{\mu}\left(\sigma+\sigma_{0}\right)=s_{\mu}(\sigma), \\
& \psi\left(\tau+\tau_{0}, 0\right)=-\psi(\tau, 0), \\
& \mathcal{C} q^{2}=-\operatorname{Im} \bar{\psi}(\tau, 0) \not(\tau) \dot{\psi}(\tau, 0), \\
& s^{2}(\sigma)=0, \\
& \$(0) \psi(\tau, 0)=0, \\
& \int_{0}^{\tau_{0}} d \tau q_{\mu}(\tau)=\int_{0}^{\sigma_{0}} d \sigma s_{\mu}(\sigma)=\Lambda_{\mu} .
\end{aligned}
$$

With the exception of Eq. (25), this is a system of algebraic relations among $q_{\mu}, s_{\mu}$, and $\psi(\tau, 0)$. Before constructing all solutions to this system explicitly, we discuss some of its general properties. First, we count the number of independent functional degrees of freedom of the system. Each of the vectors $q_{\mu}$ and $s_{\mu}$ has three real components of which two are independent by $(24)$ and $\left(8^{\prime}\right)$. $\psi(\tau, 0)$ has one complex degree of freedom by $(19)$. Apparently the system is described by four real 
and one complex degrees of freedom. However, because the equations are invariant under conformal transformations, there are two real degrees of freedom which correspond merely to changes of internal coordinates rather than to physically different states. Thus, all physically distinguishable solutions to the bubble equations are described by two real and one complex functions. These may be taken to be one real function to specify each of $q_{\mu}$ and $s_{\mu}$ and one complex function that determines $\psi(\tau, 0)$.

The charge and momentum can be simply expressed in terms of $q_{\mu}, s_{\mu}$, and $\psi(\tau, 0)$. These quantities are computed as integrals over any closed spacelike curve in the surface. Along such a curve, as $\tau$ varies from $\tau$ to $\tau+\tau_{0}$, $\sigma$ goes from $\sigma$ to $\sigma-\sigma_{0}$. We have

$$
\begin{aligned}
& \sqrt{-g} d \Sigma_{\alpha} J^{\alpha}=d \tau \bar{\psi}(\tau, 0) \mathscr{q}(\tau) \psi(\tau, 0), \\
& Q=\int_{0}^{\tau_{0}} d \tau \bar{\psi}(\tau, 0) \mathfrak{q}(\tau) \psi(\tau, 0), \\
& \sqrt{-g} d \Sigma_{\alpha} T^{\alpha \beta} \tau_{\beta}^{\mu}=\mathfrak{e}\left(q^{\mu} d \tau-s^{\mu} d \sigma\right), \\
& P^{\mu}=\mathfrak{e} \int_{\text {spacelike cut }}\left(q^{\mu} d \tau-s^{\mu} d \sigma\right)=2 \mathfrak{e} \Lambda^{\mu} .
\end{aligned}
$$

The result which is analogous to the shape degeneracy of the static bubble in three dimensions is now apparent. The energy and charge are independent of $s_{\mu} \cdot s_{\mu}$ is functionally independent of $q_{\mu}$ and $\psi(\tau, 0)$, being constrained only by the initial condition (19) and through its integral (25). Thus, the moving bubble states are degenerate over all shapes of $s_{\mu}$. As in the static case, ${ }^{2}$ the angular momentum will depend on $s_{\mu}$ through its first moment.

\section{QUANTIZED SYSTEM}

We proceed to construct the independent solutions of the algebraic Eq. ( $\left.{ }^{\prime}\right),(19),(23)$, and (24). In order to eliminate the conformal degrees of freedom, we must specify a conformal gauge by choosing one component of $q_{\mu}$ and $s_{\mu}$ each to have a definite functional dependence on $\tau$ and $\sigma$. Unfortunately, any such choice also destroys the manifest Lorentz invariance of the theory. We use the notation

$$
x^{+}=x^{0}+x^{2}, x^{-}=x^{0}-x^{2},
$$

for any vector, $x$, in Minkowski space. We specify the conformal gauge by the choice

$$
R^{+}(\tau, \sigma)=\frac{p^{+}}{2 \mathfrak{e}}(\tau+\sigma)
$$

so

$$
q^{+}=s^{+}=\frac{p^{+}}{2 \mathfrak{e}},
$$

where

$$
p^{+}=\text {constant }
$$

and

$$
\tau_{0}=\sigma_{0}=1 .
$$

A Lorentz frame and conformal gauge can always be found such that (28) holds.

Next, we make use of their periodicity to expand $q^{1}$ and $s^{1}$ in a Fourier series

$$
\begin{aligned}
q^{1}(\tau) & =\left(\frac{\pi}{\mathrm{e}}\right)^{1 / 2} \sum_{n=-\infty}^{\infty} a_{n} e^{-2 \pi i n \tau}, \\
s^{1}(\sigma) & =\left(\frac{\pi}{\mathrm{e}}\right)^{1 / 2} \sum_{n=-\infty}^{\infty} c_{n} e^{-2 \pi i n \sigma} .
\end{aligned}
$$

The coefficients $a_{n}$ and $c_{n}$ must satisfy

$$
a_{n}^{*}=a_{-n}, \quad c_{n}^{*}=c_{-n}
$$

in order to ensure that $R_{1}(\tau, \sigma)$ is real.

Condition (19) implies that $\psi(\tau, 0)$ must be of the form

$$
\psi(\tau, 0)=\left[\frac{4 \mathfrak{e}}{p^{+}} q \cdot s\right]^{-1 / 2}\left(\begin{array}{c}
1 \\
\frac{2 i \mathfrak{C}}{p^{+}} s^{1}(0)
\end{array}\right) F(\tau),
$$

where the over-all factor which multiplies the spinor has been chosen for convenience. We expand $F$ in a Fourier series:

$$
F(\tau)=\sum_{m}^{\prime} b_{m} e^{-2 \pi i m \tau}
$$

The sum in (33) is over half-odd integer $m$, so that $F$ is antiperiodic.

We can now use $\left(8^{\prime}\right)$ and (24) to compute $q^{-}$and $s^{-}$in terms of $a_{n}, c_{n}, b_{m}$.

We find

$$
\begin{aligned}
& q^{-}=\frac{4 \pi}{p^{+}} \sum_{n} \mathscr{L}_{n} e^{-2 \pi i n \tau}, \\
& s^{-}=\frac{4 \pi}{p^{+}} \sum_{n} \tilde{\mathscr{L}}_{n} e^{-2 \pi i n \sigma},
\end{aligned}
$$

where

$$
\begin{aligned}
& \mathscr{L}_{n}=\sum_{m}^{\prime}\left(m+\frac{1}{2} n\right) b_{m}^{\dagger} b_{m+n}+\frac{1}{2} \sum_{l} a_{-l} a_{l+n}, \\
& \hat{\mathscr{L}}_{n}=\frac{1}{2} \sum_{l} c_{-l} c_{l+n} .
\end{aligned}
$$

The representation given in (28)-(37) satisfies all of the algebraic constraints $\left(8^{\prime}\right),(19),(23)$, and (24). There remains the integral constraint (25). The + component of (25) is satisfied trivially: The first component of (25) requires $c_{0}=a_{0}$, an identification which we assume henceforth. The - component of the integrals in (25) gives 


$$
\mathcal{L}_{0}=\tilde{\mathscr{L}}_{0} .
$$

This is a constraint which involves all of the normal-mode amplitudes and reduces the total number of degrees of freedom by 1 . We do not use this condition to eliminate any one of the normal modes. In the quantum theory, (38) cannot be imposed as an operator condition, but rather must be imposed as a weak constraint on the physical states.

We can express the coordinate functions and the conserved charges of the bubble in terms of the normal-mode amplitudes $a_{n}, c_{n}, b_{m}$. Before doing so, it is useful to first specify the range over which $\tau$ and $\sigma$ can vary. We make this choice as follows: let

$$
\begin{aligned}
& t \equiv \sigma+\tau, \\
& \hat{\sigma} \equiv \frac{1}{2}(\tau-\sigma) .
\end{aligned}
$$

We choose

$$
\begin{aligned}
& -\infty<t<\infty, \\
& -\frac{1}{2} \leqslant \hat{\sigma} \leqslant \frac{1}{2} .
\end{aligned}
$$

This choice is useful because $t$ acts as a time, or evolution parameter, along the bubble. Unlike curves of constant $\tau$, the curves $t=$ constant are closed spacelike curves in the bubble surface.

We may write the momentum as follows:

$$
\begin{aligned}
& P^{+}=p^{+}, \\
& P^{1}=2(\pi \mathfrak{C})^{1 / 2} a_{0}, \\
& P^{-}=\frac{4 \pi \mathfrak{C}}{p^{+}}\left(\mathscr{L}_{0}+\tilde{\mathscr{L}}_{0}\right),
\end{aligned}
$$

$$
\begin{aligned}
& M^{+1}=x^{+} p^{1}-x^{1} p^{+}, \quad M^{+-}=x^{+} p^{-}-x^{-} p^{+}, \\
& M^{1-}=x^{1} p^{-}-x^{-} p^{1}+\frac{4 \pi}{p^{+}}(\pi \mathfrak{C})^{1 / 2} \sum_{n \neq 0} \frac{i}{2 \pi n}\left(a_{n} \mathscr{L}_{-n}+\mathscr{L}_{-n} a_{n}+c_{n} \tilde{\mathcal{L}}_{-n}+\tilde{L}_{-n} c_{n}\right),
\end{aligned}
$$

and the fermion number $[\mathrm{Eq} .(26)]$ is

$$
Q=\sum_{m}^{\prime} b_{m}^{\dagger} b_{m}
$$

We now have an explicit representation of all solutions to the classical bubble theory in three spacetime dimensions. In this representation, a bubble state is completely defined by giving the classical normal-mode amplitudes $a_{n}, c_{n}, b_{m}$ and the quantities $p^{+}, x^{1}, x^{-}, p^{1}$. The amplitudes which appear in $\Re^{2}$, namely, $b_{m}, a_{n}, c_{n},(n \neq 0)$, describe the internal excitations of the bubble. $P^{+}, P^{1}$, and the initial values of $x^{1}, x^{-}$give the momentum and position of the bubble.

The static states described in the preceding paper can now easily be recovered. For these states, the $\tau$ coordinate can be taken to be the time in the rest frame of the bubble. Then, in order so the mass of the bubble is

$$
\mathfrak{N}^{2}=4 \pi \mathfrak{C}\left(\mathfrak{L}_{0}+\tilde{\mathscr{L}}_{0}-a_{0}^{2}\right) .
$$

The coordinates of the surface are:

$R^{+}(\tau, \sigma)=x^{+}(\tau)$

$R^{1}(\tau, \sigma)=x^{1}(t)+\frac{i}{2(\pi \mathcal{C})^{1 / 2}} \sum_{n \neq 0} \frac{1}{n}\left(a_{n} e^{-2 \pi i n \tau}+c_{n} e^{-2 \pi i n \sigma}\right)$,

$R^{-}(\tau, \sigma)=x^{-}(t)+\frac{2 i}{p^{+}} \sum_{n \neq 0} \frac{1}{n}\left(\mathscr{L}_{n} e^{-2 \pi i n \tau}+\tilde{\mathscr{L}}_{n} e^{-2 \pi i n \sigma}\right)$,

where

$$
\begin{aligned}
& x^{+}(t)=\frac{p^{+}}{2 \mathfrak{e}} t=\frac{p^{+}}{2 \mathfrak{e}}(\tau+\sigma), \\
& x^{1}(t)=\frac{p^{1}}{2 \mathfrak{e}} t+\bar{x}^{1}, \\
& x^{-}(t)=\frac{p^{-}}{2 \mathfrak{e}} t+\bar{x}^{-} .
\end{aligned}
$$

$\bar{x}^{-}$and $\bar{x}^{1}$ are constants of integrations. The angular momenta are for the bubble to be static, we must take $a_{n}=0$ for all $n$. A $Q=1$ positive-energy state of the quark field corresponds to $b_{m}=1$ for some value of $m>0 . c_{n}$ can be chosen arbitrarily, subject only to the constraint (38). $p^{+}$must be chosen to be $\mathfrak{M}$ so that the bubble will be at rest. The mass of such a state is

$$
\mathfrak{T}^{2}=4 \pi \mathfrak{C}\left(\mathfrak{L}_{0}+\tilde{\mathfrak{L}}_{0}\right)=8 \pi \mathfrak{C} m
$$

in agreement with the calculations of the preceding paper.

Although we have treated the case of only one quark field, it is straightforward to extend the solution to the case where there are $N$ species of quarks trapped on the bubble, where $i \hbar \psi_{j}=\psi_{j}$. We simply remark that Eq. (36) then becomes

$$
\mathscr{L}_{n}=\sum_{j=1}^{N} \sum_{m}^{\prime}\left(m+\frac{1}{2} n\right) b_{j m}^{\dagger} b_{j, m+n}+\frac{1}{2} \sum_{l} a_{-l} a_{l+n},
$$


and the fermion number $Q$ also extends to a sum over all species.

Because all classical solutions of the theory are available to us, we can construct the quantum theory of the bubble explicitly. The noncanonical quantization of the bubble which we present is neither manifestly gauge nor Lorentz invariant. Its Lorentz invariance must be explicitly demonstrated.

We would expect any quantum theory of the bubble to induce simple commutation relations between the normal-mode amplitudes of the classical theory. In the following discussion, we shall quantize the bubble by introducing a set of fundamental commutation relations among the independent normal-mode amplitudes. Our guide in choosing these commutation relations will be the requirement that the canonical Poincaré and charge operators have the correct algebra.

We require, specifically, that the commutation relations guarantee

(i) that each quark has fermion number 1 ,

$$
\left[Q, b_{j m}^{\dagger}\right]=b_{j m}^{\dagger}, \quad m>0,
$$

(ii) that the canonical momentum and angular momentum operators, (39) and (42), satisfy the correct Poincaré algebra,

(iii) that the constraint (38) imposed weakly on states is consistent with Poincare invariance, that is, that $\mathscr{L}_{0}-\tilde{\mathscr{L}}_{0}$ commute with all the Poincare generators.

Rather than outline the derivation of the correct commutation relations from the requirements (i), (ii), and (iii), we will begin with the fundamental commutation relations and sketch the verification of the operator algebra.

We take the commutation relations of the normal-mode amplitudes to be

$$
\begin{aligned}
& {\left[a_{n,} a_{l}\right]=n \delta_{n,-l},} \\
& {\left[c_{n}, c_{l}\right]=n \delta_{n,-l},} \\
& \left\{b_{m}, b_{m^{\prime}}^{\dagger}\right\}=\delta_{m, m^{\prime}},
\end{aligned}
$$

with all other commutators of the independent modes vanishing. We shall regard $a_{n}, c_{n}(n>0)$ as annihilation operators and $a_{n}^{\dagger}=a_{-n}, c_{n}^{\dagger}=c_{-n}$ as the creation operators. The vacuum $|0\rangle$ is defined by

$$
\begin{aligned}
& a_{n}|0\rangle=0, \\
& b_{m}|0\rangle=0, m, n>0 \\
& d_{m}|0\rangle=0, \\
& c_{n}|0\rangle=0,
\end{aligned}
$$

where $d_{m}=b_{-m}^{\dagger}$ are the annihilation operators for the antiquark modes, $d_{m}^{\dagger}=b_{-m}$ creates an antiquark, and $b_{m}, b_{m}^{\dagger}$ are the annihilation and creation operators for a quark, respectively. A positive-energy spectrum is thus achieved. The dependent variables $q^{-}$and $s^{-}$are given in terms of these by Eqs. (34) and (35). There is an ambiguity in ordering the operators in $\mathscr{L}_{0}$ and $\tilde{\mathscr{L}}_{0}$. Let

$$
p^{-}=\frac{4 \pi \mathfrak{e}}{p^{+}}\left[\left(\mathcal{L}_{0}-\Lambda\right)+\left(\tilde{\mathscr{L}}_{0}-\bar{\Lambda}\right)\right]
$$

where by $\mathscr{L}_{0}, \tilde{L}_{0}$ we now mean the normal-ordered expressions with respect to the vacuum defined by Eq. (46). $\Lambda$ and $\bar{\Lambda}$ are constants, for the moment arbitrary. With this definition, Eqs. (44) and (45) give

$$
\begin{aligned}
& {\left[\mathscr{L}_{n}, \mathcal{L}_{m}\right]=(n-m) \mathscr{L}_{n+m}+\delta_{n,-m}\left(n^{3}-n\right) \frac{1}{12}(N+1)} \\
& {\left[\tilde{\mathscr{L}}_{n}, \tilde{\mathscr{L}}_{m}\right]=(n-m) \tilde{\mathscr{L}}_{n+m}+\delta_{n,-m}\left(n^{3}-n\right) \frac{1}{12},}
\end{aligned}
$$

where $N$ is the number of species of quarks. After normal ordering, $Q$ becomes

$$
Q=\sum_{j=1}^{N} Q_{j}=\sum_{j=1}^{N} \sum_{m>0}^{\prime}\left(b_{j m}^{\dagger} b_{j m}-d_{j m}^{\dagger} d_{j m}\right),
$$

and the correct charge commutator follows:

$$
\begin{aligned}
& {\left[Q_{i}, b_{j m}^{\dagger}\right]=b_{j m}^{\dagger} \delta_{i j},} \\
& {\left[Q_{i}, d_{j m}^{\dagger}\right]=-d_{j m}^{\dagger} \delta_{i j} .}
\end{aligned}
$$

We also have

$$
\begin{aligned}
& {\left[\mathcal{L}_{n}, a_{l}\right]=-l a_{l+n},} \\
& {\left[\tilde{\mathcal{L}}_{n}, c_{l}\right]=-l c_{l+n},} \\
& {\left[\mathcal{L}_{n}, b_{m}\right]=-\left(m+\frac{1}{2} n\right) b_{m+n},} \\
& {\left[\mathcal{L}_{n}, b_{m}^{\dagger}\right]=\left(m-\frac{1}{2} n\right) b_{m-n}^{\dagger},}
\end{aligned}
$$

where the quark-species index is suppressed.

We must also define commutators involving the momenta $P^{\mu}$ and coordinates $x_{\mu}(t)$. These are determined by the requirement that $P^{\mu}$ generate translations of the bubble. The commutation relations must be such that

$$
\left[\delta G, R^{\mu}\right]=i \delta R^{\mu},
$$

where $\delta G=P^{\mu} \delta a_{\mu}$ is the generator of the infinitesimal translation $\delta a_{\mu}$ and $\delta R^{\mu}$ is the infinitesimal shift in $R^{\mu}$. The representation of the bubble surface we have chosen is not Poincaré invariant. In order to maintain the gauge condition

$$
R^{+}(\tau, \sigma)=x^{+}(t)=\frac{p^{+}}{2 \mathfrak{e}} t
$$

We must perform a conformal transformation along with the translation:

$$
t \rightarrow t-\frac{2 \mathfrak{e}}{p^{+}} \delta a^{+} .
$$

Thus, the total shift $\delta R^{\mu}$ is

$$
\delta R^{\mu}=\delta a^{\mu}-\frac{2 \mathfrak{e}}{p^{+}} \frac{\partial R^{\mu}}{\partial t} \delta a^{+} .
$$


Through (52) this gives the commutation relations of the momenta $P^{\mu}$ and the coordinate variables $x^{\mu}(t)$. The nonvanishing commutators are

$$
\begin{aligned}
& {\left[P^{1}, x^{1}\right]=-i,} \\
& {\left[P^{-}, x^{1}\right]=-\frac{2 i P^{1}}{p^{+}},} \\
& {\left[p^{+}, x^{-}\right]=2 i,} \\
& {\left[P^{-}, x^{-}\right]=-\frac{2 i P^{-}}{p^{+}} .}
\end{aligned}
$$

In deriving (53), we note that the relations (50), (51) have been used. For example,

$$
\frac{\partial}{\partial t}\left(\sum_{n} a_{n} e^{-2 \pi i n \tau}\right)=2 \pi i\left[\mathscr{L}_{0}, \sum_{n} a_{n} e^{-2 \pi i n \tau}\right] .
$$

We take the classical expressions (42) as the definition of the Lorentz generators, with the additional assumption that products of noncommuting operators in (42) are to be Hermitian symmetrized. For example, we take

$$
M^{+-}=x^{+} p^{-}-\frac{1}{2}\left(x^{-} p^{+}+p^{+} x^{-}\right) \text {. }
$$

It is straightforward to show that the Poincaré algebra is satisfied, both formally and for the normal-ordered operators.

From the commutation relations (46) and (47) it can easily be verified that $\left(\mathscr{L}_{0}-\tilde{\mathscr{L}}_{0}\right)$ commutes with the charge and with all of Poincaré generators. Thus, the constraint that physical states obey

$$
\left.\left(\mathscr{L}_{0}-\tilde{\mathscr{L}}_{0}-\Lambda+\bar{\Lambda}\right) \mid \text { physical }\right\rangle=0
$$

is consistent with Poincare invariance of the theory.

We may also introduce an operator which corresponds to the spin of the bubble:

$$
\begin{aligned}
& W=\frac{1}{2} \epsilon_{\mu \nu \lambda} P^{\mu} M^{\nu \lambda} \\
& =-4 \pi(\pi \mathfrak{C})^{1 / 2} \sum_{n \neq 0} \frac{i}{2 \pi n}\left(a_{n} \mathscr{L}_{-n}+\mathscr{L}_{-n} a_{n}\right. \\
& \left.\quad+c_{n} \tilde{\mathscr{L}}_{-n}+\tilde{\mathscr{L}}_{-n} c_{n}\right) .
\end{aligned}
$$

Classically, in the rest frame of the bubble,

$$
W=P^{0} M^{12}=\mathfrak{T}^{\mathrm{J}} J_{3} \text {. }
$$

$W$ commutes with both $Q$ and $\mathfrak{M}^{2}$, and is unchanged by normal ordering.

We have now exhibited a self-consistent quantized operator algebra corresponding to the bubble theory in three spacetime dimensions. There are conserved fermion numbers, $Q_{j}$, and the theory has been shown to be Poincaré invariant.

\section{THE SPECTRUM}

To study the physical states of the theory, we encounter the ambiguities associated with the nor- mal orderings of operators. In terms of the constants $\Lambda$ and $\bar{\Lambda}$, the normal-ordered mass operator is

$$
\mathfrak{M}^{2}=4 \pi \mathcal{C}\left(\mathscr{L}_{0}+\tilde{\mathscr{L}}_{0}-a_{0}{ }^{2}-\Lambda-\bar{\Lambda}\right),
$$

and the weak constraint on any state $\left|\Psi_{p}\right\rangle$ is given by Eq. (54), where

$$
\begin{aligned}
& \mathscr{L}_{0}=\sum_{n>0} a_{n}^{\dagger} a_{n}+\frac{a_{0}^{2}}{2}+\sum_{j=1}^{N} \sum_{m=1 / 2}^{\infty} ' m\left(b_{j m}^{\dagger} b_{j m}+d_{j m}^{\dagger} d_{j m}\right), \\
& \tilde{\mathscr{L}}_{0}=\frac{a_{0}^{2}}{2}+\sum_{n>0} c_{n}^{\dagger} c_{n}
\end{aligned}
$$

It is clear that states of definite particle number are eigenstates of $Q, \mathscr{L}_{0}$, and $\tilde{\mathscr{L}}_{0}$. All states lie on straight Regge trajectories with Regge slope

$$
\alpha^{\prime}=\frac{1}{8 \pi \mathfrak{e}} \text { 。 }
$$

The intercept of the leading trajectory is $\Lambda+\bar{\Lambda}$.

That the Regge trajectories are straight is not surprising because the model is that of a two-dimensional object characterized by a single dimensional parameter. Curved trajectories probably require a second dimensional parameter to determine its curvature characteristic。

The Hilbert space of the theory becomes well defined only after we have assigned finite values to the constants

$$
\alpha_{0}=\Lambda+\bar{\Lambda}, \quad \Delta=\bar{\Lambda}-\Lambda .
$$

The actual values we choose for $\alpha_{0}$ and $\Delta$ are arbitrary - they are unconstrained by the operator algebra. Further, no matter what the value of $\alpha_{0}$, the condition (54) will place severe restrictions on the spectrum. $\tilde{\mathscr{L}}_{0}-a_{0}^{2} / 2$ has only integral eigenvalues. $\mathscr{L}_{0}-a_{0}{ }^{2} / 2$ has integral eigenvalues if $Q$ is even and half-odd integral eigenvalues if $Q$ is odd. Therefore, if $\Delta$ is an integer, we form only states of even fermion number. If $\Delta$ is a halfodd integer, all states must have odd fermion number.

We shall discuss only two of the infinite number of possible choices of $\alpha_{0}$ and $\Delta$. Our guide in the selection of $\alpha_{0}$ and $\Delta$ will be the classical theory. The operators $\mathcal{L}_{0}$ and $\tilde{L}_{0}$ appear on the equal footing in the mass operator. $\mathscr{L}_{0}$ is the contribution of the fermion and the " $a$ " surface excitations to the bubble energy; $\tilde{\mathscr{L}}_{0}$ is the contribution of the " $c$ " surface excitations. In the classical theory, $\mathscr{L}_{0}$ and $\tilde{\mathscr{L}}_{0}$ contribute equally to the mass, and we can write

$$
\mathfrak{M}^{2}=4 \pi \mathfrak{e}\left(\mathscr{L}_{0}-a_{0}^{2} / 2\right) .
$$

In the case of the static classical bubble, there are no " $a$ " excitations, and the " $c$ " excitations are forced to be nonvanishing in the presence of any fermions to satisfy the constraint (38). We maintain these 
features in any quantum theory defined by choosing $\alpha_{0}=\Delta \geqslant 0$. We will consider the spectrum of the simplest such cases:

fermionic states, $\alpha_{0}=\Delta=\frac{1}{2}$,

bosonic states, $\alpha_{0}=\Delta=0$.

In case of Eq. (59) the mass levels of the $Q=1$ states are exactly those of the classical static theory:

$$
\mathfrak{M}^{2}=8 \pi \mathfrak{e} l_{0}
$$

where $l_{0}$ is the eigenvalue of $\mathscr{L}_{0}-a_{0}^{2} / 2$, a positive half-odd integer. We see that the degeneracy of each of these levels is finite. Table I lists the degenerate states comprising the first few levels for a single type of quark.

The breaking of the semiclassical degeneracy of levels over all bubble shapes is an easily understood quantum effect. Classically, the only constraint on $\left\{c_{n}\right\}$ is (38). Because each classical normal-mode coefficient can take on a continuum of values, this constraint can be satisfied by an uncountably infinite number of combinations of $\left\{c_{n}\right\}$. In the quantum theory, however, the energy associated with each mode becomes discrete, so there are only a finite number of combinations of occupation numbers which sum to any given finite energy, $l_{0}+\frac{1}{2}$. In the quantum theory, the softness of the bubble becomes apparent in two ways. First, the size of the fluctuations in the surface coordinates is always comparable to the size of the bubble itself; a result which follows from the absence of any dimensionless parameters which might serve to set an independent scale for the size of fluctuations. Also, as simple combinatorics indicates, as the excitation energy increases, the degeneracy of the levels increases exponentially as $\exp [(\Re / / \sqrt{\mathrm{e}})$ (constant)].

The spin operator, $W$, can be diagonalized si-

TABLE I. The low-lying single fermionic states of the three-dimensional bubble, where $\alpha_{0}=\Delta=\frac{1}{2}, l_{0}$ is the eigenvalue of $\mathscr{L}_{0}-a_{0}{ }^{2} / 2, \tilde{l}_{0}$ is the eigenvalue of $\tilde{L}_{0}-a_{0}^{2} / 2$, $\omega$ is the eigenvalue of $W /(8 \pi \mathfrak{C})^{1 / 2}$, and $\mathfrak{T}^{2}=8 \pi \mathfrak{C} l_{0}$.

\begin{tabular}{cccc}
\hline \hline$l_{0}$ & $\tilde{l}_{0}$ & $\omega$ & State vector \\
\hline$\frac{1}{2}$ & 1 & 0 & $b_{1 / 2}^{\dagger} c_{1}^{\dagger}|0\rangle$ \\
$\frac{3}{2}$ & 2 & $-\frac{5}{\sqrt{2}}$ & $\frac{1}{2 \sqrt{2}}\left(b_{3 / 2}^{\dagger}-i b_{1 / 2}^{\dagger} a_{1}^{\dagger}\right)\left[c_{2}^{\dagger}-i\left(c_{1}^{\dagger}\right)^{2}\right]|0\rangle$ \\
& & $-\frac{1}{\sqrt{2}}$ & $\frac{1}{2 \sqrt{2}}\left(b_{3 / 2}^{\dagger}+i b_{1 / 2}^{\dagger} a_{1}^{\dagger}\right)\left[c_{2}^{\dagger}-i\left(c_{1}^{\dagger}\right)^{2}\right]|0\rangle$ \\
& & $+\frac{1}{\sqrt{2}}$ & $\frac{1}{2 \sqrt{2}}\left(b_{3 / 2}^{\dagger}-i b_{1 / 2}^{\dagger} a_{1}^{\dagger}\right)\left[c_{2}^{\dagger}+i\left(c_{1}^{\dagger}\right)^{2}\right]|0\rangle$ \\
& $+\frac{5}{\sqrt{2}}$ & $\frac{1}{2 \sqrt{2}}\left(b_{3 / 2}^{\dagger}+i b_{1 / 2}^{\dagger} a_{1}^{\dagger}\right)\left[c_{2}^{\dagger}+i\left(c_{1}^{\dagger}\right)^{2}\right]|0\rangle$ \\
\hline \hline
\end{tabular}

multaneously with $Q$ and $\mathfrak{M}^{2}$. Table I also indicates the eigenstates and eigenvalues of $W$ among the three lowest levels of the $Q=1$ "fermionic" spectrum. Because of the normal-ordering ambiguities, we have not been able to relate the eigenvalues of $W$ directly to a "physical" spin of the bubble. The source of the difficulty is precisely the same as that which leaves $\alpha_{0}$ and $\Delta$ undetermined. There are no nontrivial commutation relations between $W$ and other operators of the theory which might serve to fix the scale of $W$ when the theory is made finite by normal ordering. More concretely, we observe that classically the spin is given by

$$
J_{3}=\frac{W}{\mathfrak{T}} \text {. }
$$

In the normal-ordered quantum theory we have no analogous result.

If we assume color $\mathrm{SU}(3)$ so that baryons are formed from three quarks in a color singlet state, then the lowest baryonic state is $\left[c_{2}^{\dagger} \pm\left(c_{1}^{\dagger}\right)^{2}\right]$

$\times b_{i 1 / 2}^{\dagger} b_{j 1 / 2}^{\dagger} b_{k 1 / 2}^{\dagger}|0\rangle$, where $i, j, k$ designate different color quarks.

Next, we consider the spectrum of "mesons," taking $\alpha_{0}=\Delta=0$. The mass levels are

$$
\begin{aligned}
& \mathfrak{N}^{2}=8 \pi \mathfrak{C} l_{0}, \\
& l_{0}=0,1,2, \ldots .
\end{aligned}
$$

The states corresponding to the first three meson levels are given in Table II, along with the corresponding eigenvalues and eigenstates of $W$. The meson spectrum has many of the qualitative features of the fermionic spectrum. We remark upon only two special aspects of it. First, the lowest state is the state that we have called the vacuum. This vacuum is not, then, the usual vacuum state of a multiparticle theory. It is, rather, the low est-lying state in the spectrum of a single system with many possible internal excitations. With the choice of normal -ordering parameters we have made, the vacuum is a massless bubble state, and has no classical analog. Second, we note that the meson spectrum contains states which correspond to bubbles containing no quarks at all. These are purely surface excitations, and are analogous to the excitations of a closed dual string in threedimensional Minkowski space.

In the BCDWY model with color SU(3), there is a problem where color-singlet states tend to condense, for example, $N$ mesons each with a quarkantiquark pair have higher energy than a singlemeson state with the same quark-antiquark pairs. Condensation may be avoided by certain choices of the intercept parameter $\alpha_{0}$. For example, in the bosonic sector with $\alpha_{0}=\Delta=1$, the vacuum state has negative (mass) ${ }^{2}$ and is a tachyon, the lowest $q \bar{q}$ 
TABLE II. The low-lying "meson" states of the three-dimensional bubble, where $\alpha_{0}=\Delta=0$ and $c_{ \pm}^{\dagger} \equiv \frac{1}{2}\left[c_{2}^{\dagger} \pm i\left(c_{1}^{\dagger}\right)^{2}\right]$.

\begin{tabular}{cccc}
\hline \hline$l_{0}$ & $\tilde{l}_{0}$ & $\omega$ & State vector \\
\hline 0 & 0 & 0 & $|0\rangle$ \\
1 & 1 & 0 & $a_{1}^{\dagger} c_{1}^{\dagger}|0\rangle$ \\
& & 0 & $b_{1 / 2}^{\dagger} d_{1 / 2}^{\dagger} c_{1}^{\dagger}|0\rangle$ \\
2 & 2 & $\pm \frac{3}{\sqrt{2}}-\sqrt{5}$ & $c_{ \pm}^{\dagger}\left[\frac{i}{2} a_{2}^{\dagger}+\frac{3}{\sqrt{40}}\left(a_{1}^{\dagger}\right)^{2}+\frac{1}{\sqrt{40}}\left(b_{1 / 2}^{\dagger} d_{3 / 2}^{\dagger}+d_{1 / 2}^{\dagger} b_{3 / 2}^{\dagger}\right)\right]|0\rangle$ \\
& $\pm \frac{3}{\sqrt{2}}-2$ & $c_{ \pm}^{\dagger}\left[-\frac{i}{\sqrt{2}} b_{1 / 2}^{\dagger} d_{1 / 2}^{\dagger} a_{1}^{\dagger}+\frac{1}{2}\left(b_{1 / 2}^{\dagger} d_{3 / 2}^{\dagger}-d_{1 / 2}^{\dagger} b_{3 / 2}^{\dagger}\right)\right]|0\rangle$ \\
& $\pm \frac{3}{\sqrt{2}}$ & $c_{ \pm}^{\dagger}\left[-\frac{1}{\sqrt{20}}\left(a_{1}^{\dagger}\right)^{2}+\frac{3}{\sqrt{20}}\left(b_{1 / 2}^{\dagger} d_{3 / 2}^{\dagger}+d_{1 / 2}^{\dagger} b_{3 / 2}^{\dagger}\right)\right]|0\rangle$ \\
& $\pm \frac{3}{\sqrt{2}}+2$ & $c_{ \pm}^{\dagger}\left[\frac{i}{\sqrt{2}} b_{1 / 2}^{\dagger} d_{1 / 2}^{\dagger} a_{1}^{\dagger}+\frac{1}{2}\left(b_{1 / 2}^{\dagger} d_{3 / 2}^{\dagger}-d_{1 / 2}^{\dagger} b_{3 / 2}^{\dagger}\right)\right]|0\rangle$ \\
& $\pm \frac{3}{\sqrt{2}}+\sqrt{5}$ & $c_{ \pm}^{\dagger}\left[\frac{i}{2} a_{2}^{\dagger}-\frac{3}{\sqrt{40}}\left(a_{1}^{\dagger}\right)^{2}-\frac{1}{\sqrt{40}}\left(b_{1 / 2}^{\dagger} d_{3 / 2}^{\dagger}+d_{1 / 2}^{\dagger} b_{3 / 2}^{\dagger}\right)\right]|0\rangle$ \\
\hline
\end{tabular}

state is massless, and higher $(q \bar{q} q \bar{q})$ exotics are energetically unstable with respect to decay into the lowest $q \bar{q}$ states. In the absence of a theory encompassing interactions, $\alpha_{0}$ and $\Delta$ are completely free.

The quantum theory we have constructed is the theory of a single particle, which has many possible internal excitations. In a theory which is to reflect more accurately the properties of the real world, we must have mechanisms by which these particles scatter and are created and annihilated. One might hope that, in analogy with the string theory, such mechanisms are already implicit in the formulation of the bubble model.

An attractive classical picture of bubble-bubble interactions is that bubbles interact with each other by fusing or fissioning when their surfaces touch. Such a picture is the analogy in bubble theory of the fission and fusion of MIT bags ${ }^{4}$ or of dual strings. Generally, string, bubble, and bag theories have classical solutions which correspond to such processes. For the bubble, such a solution would be characterized by the existence of surface singularities at which the evolution of the classical bubble becomes indeterminate. Of the possible solutions for the evolution of the system is one in which a single bubble state emerges and others which correspond to the formation of new bubbles.

Mandelstam ${ }^{5}$ has shown, in the open-string mod$\mathrm{el},{ }^{3}$ that string-string scattering amplitudes can be computed via a path-integral method which includes paths corresponding to the classical fission and fusion of strings. It seems hopeful that such a procedure might be formulated for the bubble case in three spacetime dimensions.

In principle, at least, we are in a position to com- pute the form factor of the bubble in three dimensions. The operator whose matrix elements give the form factor is the Fourier transform of the current density,

$J^{\mu}(q)=: \int d^{2} u \sqrt{-g} \exp \left[-q_{\mu} R^{\mu}\left(u^{\alpha}\right)\right] \bar{\psi}\left(u^{\alpha}\right) \gamma^{\mu} \psi\left(u^{\alpha}\right):$.

The normal-ordering difficulties which arise in any attempt to evaluate a finite matrix element of this operator are nontrivial.

Since in real life, states of the empty bubble (i.e., states with bosonic oscillators only) are not observed, one may hope that bubble scattering vertices can be constructed in such a way that all empty bubble modes do not couple. We do not know of any other way to exclude the pure bosonic modes from the spectrum.

We observe that in the absence of fermion field in the bubble action, the dual string emerges. It follows that the energy-momentum stress tensor $T^{\alpha \beta}$ simply becomes the metric tensor $g^{\alpha \beta}$, and the solution reduces to that of Ref. 3. (We choose $g^{\alpha \beta}$ off-diagonal instead of diagonal, this simply means our parameters $\tau$ and $\sigma$ are the light-cone version of the $\tau$ and $\sigma$ in ref 3.)

The quark field here is a physical quark field in that it obeys a Dirac equation in the Minkowski space. It is a conformal scalar in the two-dimensional $(\tau, \sigma)$ subspace. Hence, it is different from the Neveu-Schwarz-Ramond model $^{6}$ where the fermion field is a spinor in the two-dimensional $(\tau, \sigma)$ space, and is a two-component vector field in the physical Minkowski space, the timelike component of which has the wrong metric. This has to be eliminated to avoid ghosts, which is achieved by having extra dependence among the dynamical var- 
iables. Actually this quark-binding bubble is closely related to the Bardakci-Halpern model. ${ }^{7}$

Topologically we classify different geometrically constructed extended-hadron models into three types: (1) string, a two-dimensional subspace embedded in an $n$-dimensional Minkowski space, (2) bubble, a $(n-1)$-dimensional hypersurface embedded in an $n$-dimensional Minkowski space (here one can also include membranelike model), (3) bag, a finite volume in an $n$-dimensional Minkowski space.

Thus, in three-dimensional Minkowski space, a bubble and a string are equivalent. In two-dimensional Minkowski space, a string and a bag are equivalent.

The three-dimensional bubble (or string) lacks sufficient resemblance to the real world; because there is but one component of angular momentum and hence no algebraic constraints on the normalordering terms, the spectrum of states remains ambiguous (i.e., values for $\Lambda, \bar{\Lambda}$ ).

To extend to physical Minkowski space, it is not clear which is the proper generalization. The bubble is investigated in Ref. 2. The string is investigated in Ref. 8. The latter offers the hope of being solved completely. Only a confrontation with experiments can tell which is closer to nature: bag, bubble, or string, or none of them.

The quantization of the bubble in this work is noncovariant. It will be interesting to carry out its covariant quantization. A knowledge of Ref. 7 may suggest an approach to covariant quantization for the case with four-component Dirac particles [see Eq. (6)].
*Work supported by the U.S. Energy Research and Development Administration.

†Work supported in part by the Fannie and John Hertz Foundation.

${ }^{1}$ W. A. Bardeen, M. S. Chanowitz, S. D. Drell, M. Weinstein, and T.-M. Yan, Phys. Rev. D 11, 1094 (1974).

${ }^{2}$ R. C. Giles, preceding paper, Phys. Rev. D $\underline{13}, 1670$ (1976).

${ }^{3}$ Y. Nambu, Lectures at the Copenhagen Summer Symposium, 1970 (unpublished); P. Goddard, J. Goldstone, C. Rebbi, and C. B. Thorn, Nucl. Phys. B56, 109
(1973). Earlier references can be found here.

${ }^{4}$ A. Chodos, R. L. Jaffe, K. Johnson, C. B. Thorn, and V. F. Weisskopf, Phys. Rev. D 9 , 3471 (1974).

${ }^{5}$ S. Mandelstam, Nucl. Phys. B64, 205 (1975).

${ }^{6}$ A. Neveu and J. H. Schwarz, Nucl. Phys. B31, 86

(1971); P. Ramond, Phys. Rev. D 3, 2493 (1971). See

also Y. Iwasaki and K. Kikkawa, ibid . 8, 440 (1973).

${ }^{7} \mathrm{~K}$. Bardakci and M. B. Halpern, Phys. Rev. D $\underline{3}, 2493$ (1971).

${ }^{8}$ S.-H. H. Tye, SLAC Report No. 1673, 1975 (unpublished). 